\title{
Intervenção PRAdA: Efeitos no Peso Induzidos por Proposta de Alteração de Comportamento Alimentar em Hipertensos Medicados Nativos Portugueses e Imigrantes de Origem Africana
}

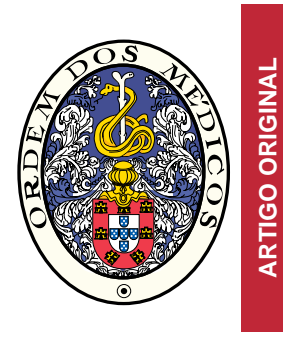

\author{
PRAdA Intervention: Effects on Weight of Dietary Intervention among Treated \\ Hypertensive Portuguese Natives and African Immigrants
}

Catarina PEIXOTO ${ }^{1}$, Gisela CARRILHO ${ }^{1}$, Violeta ALARCÃO ${ }^{1}$, Filipa GUERRA ${ }^{1}$, Rui SIMÕES ${ }^{1}$, Milene FERNANDES ${ }^{1}$, Paulo NICOLA ${ }^{1}$, Sofia GUIOMAR ${ }^{1,2}$, Paulo NOGUEIRA ${ }^{1}$, Evangelista ROCHA ${ }^{1}$

Acta Med Port 2014 May-Jun;27(3):331-341

\section{RESUMO}

Introdução: Perdas de peso melhoram o controlo da tensão arterial em indivíduos hipertensos. As intervenções comportamentais para a perda de peso parecem ser menos eficazes nos imigrantes de origem africana (IOA). Os principais objetivos foram avaliar os efeitos no peso de uma intervenção alimentar em hipertensos nativos de origem portugueses (NOP) e IOA; identificar fatores de sucesso na perda de peso; avaliar a evolução dos conhecimentos e adesão às recomendações alimentares.

Material e Métodos: Foram selecionados aleatoriamente hipertensos medicados seguidos nos cuidados de saúde primários para um estudo de duas fases, observacional (15 meses) e de intervenção comportamental (seis meses). Os participantes foram divididos em dois grupos: IOA e NOP. Foram dadas recomendações alimentares e de estilos de vida em sessões individuais presenciais e telefónicas.

Resultados: Dos 110 participantes, com IMC médio de $31,6 \pm 3,7 \mathrm{~kg} / \mathrm{m}^{2}$ e idade média $61,3 \pm 10,0$ anos, 60 eram imigrantes. Durante a intervenção os NOP perderam $1,4 \pm 2,7 \%$ do peso e os IOA $0,8 \pm 3,6 \%$, sendo a perda superior comparativamente com a fase observacional. Ser do sexo masculino e consumir pelo menos duas porções de lacticínios magros por dia associou-se a maior perda de peso durante a intervenção, independentemente da idade e origem. Houve um aumento significativo no número de recomendações dietéticas conhecidas e cumpridas, com os NOP a cumprirem em média mais recomendações.

Discussão: A intervenção foi bem sucedida relativamente à perda de peso, especialmente no grupo dos NOP, confirmando outros estudos.

Conclusão: A intervenção aumentou os conhecimentos e adesão às recomendações, realçando a pertinência da educação alimentar, culturalmente adaptada, nos cuidados de saúde primários.

Palavras-chave: África; Emigrantes e Imigrantes; Estudos de Intervenção; Hábitos Alimentares; Hipertensão; Perda de Peso; Portugal.

\section{ABSTRACT}

Introduction: Blood pressure is significantly improved with weight loss. Behavioral interventions for weight loss seem to be less successful in African immigrants. Our main aims were to assess the effect of a dietary and lifestyle intervention on weight among hypertensive Portuguese natives and immigrants and to identify success factors for weight loss, and also to evaluate changes in knowledge and compliance with food recommendations.

Material and Methods: Hypertensive medicated patients followed in primary care setting were randomly enrolled in a two phase study, observational (15-months) and behavioral intervention (six months). Participants were divided in two groups: immigrants from African Countries of Portuguese Official Language and Portuguese natives. Participants were given dietary and life styles recommendations in individual face-to-face and telephone sessions.

Results: Of 110 participants with a mean BMI of $31.6 \pm 3.7 \mathrm{Kg} / \mathrm{m}^{2}, 60$ were immigrants. The number of dietary recommendations known and followed at the end was significantly greater than at baseline; however natives performed a greater number of recommendations. Weight loss during intervention was in average $1.4 \pm 2.7 \%$ in natives and $0.8 \pm 3.6 \%$ in immigrants and was greater than in the observational period. Being male and consuming more than 2 servings of low-fat dairy products/day was associated with higher weight loss, independently of age and ethnicity.

Discussion: In general the proposed intervention was efficacious especially in Portuguese natives, confirming other studies.

Conclusion: The intervention increased knowledge and adherence to recommendations, highlighting the relevance of nutrition education, culturally adapted in primary care.

Keywords: Africa; Emigrants and Immigrants; Intervention Studies; Food Habits; Hypertension; Weight Loss; Portugal.

\section{INTRODUÇÃo}

A redução do peso para indivíduos com excesso de peso ou obesos, o baixo consumo de sódio, moderação no consumo de álcool, dieta variada e equilibrada com consumo de frutas, vegetais e laticínios magros, ${ }^{1}$ a cessação tabágica ${ }^{2}$ e a prática de atividade física ${ }^{3}$ encontram-se entre as modificações do estilo de vida que efetivamente reduzem a pressão arterial (PA). ${ }^{4} \mathrm{~A}$ alimentação é por isso um fator determinante na diminuição do risco cardiovascular em doentes hipertensos. ${ }^{1}$

Vários fatores de risco cardiovasculares, entre os quais a hipertensão (HTA), afetam de forma desigual negros e caucasianos. ${ }^{5}$ Estudos internacionais indicam que os ne-

1. Unidade de Epidemiologia. Instituto de Medicina Preventiva. Faculdade de Medicina de Lisboa. Lisboa. Portugal.

2. Instituto Nacional de Saúde Doutor Ricardo Jorge. Lisboa. Portugal.

Recebido: 10 de Setembro de 2013 - Aceite: 24 de Janeiro de 2014 | Copyright $\odot$ Ordem dos Médicos 2014 
gros, quando comparados com os restantes grupos étnicos, são mais vulneráveis aos efeitos da HTA, apresentando uma maior prevalência, gravidade e risco de desenvolver HTA precocemente. ${ }^{6}$ Quando avaliadas as intervenções de programas comportamentais de perda de peso, estas parecem ser menos eficazes nos negros do que nos caucasianos. ${ }^{7,8}$

Os resultados do estudo Trial of Nonpharmacologic Interventions in the Elderly (TONE), com hipertensos medicados, divididos em duas coortes, brancos e negros com excesso de peso, apontam uma perda de peso (média), no final de seis meses, de 2,7 kg nos negros e de $5,9 \mathrm{~kg}$ nos caucasianos. ${ }^{7}$ Outros estudos com intervenções similares reportam perdas de peso inferiores nas mulheres negras comparativamente aos homens negros e à etnia caucasiana. ${ }^{8,9}$

Em Portugal, dos 341313 imigrantes em situação regular, 21,6\% são oriundos dos Países Africanos de Língua Oficial Portuguesa (PALOP). ${ }^{10}$ Segundo os dados do quarto Inquérito Nacional de Saúde 2005/06, a HTA é uma das doenças crónicas mais frequentemente autorreportadas entre as comunidades imigrantes $(13,1 \%$ para imigrantes e 18,6\% portugueses). ${ }^{11}$ Em Portugal, desconhecem-se estudos que comparem a eficácia de intervenções alimentares e de estilo de vida na perda de peso entre nativos de origem portuguesa (NOP) e imigrantes de origem africana (IOA) e os dados de prevalência do excesso de peso em minorias étnicas são escassos.

Os determinantes socioeconómicos e a dificuldade em aceder e usar os serviços de saúde disponíveis têm ganho especial relevo na tentativa de compreensão da relação entre imigração e vulnerabilidade em saúde. ${ }^{12-14}$

Estudos realizados em Portugal com vista à perda de peso confirmam como preditores de sucesso na perda de peso a curto prazo o número de tentativas de perda de peso anteriores, a auto motivação, a perceção da imagem corporal e foco inicial na dieta enquanto a mudança nos fatores motivacionais intrínsecos (por exemplo, prazer em fazer exercício) desempenha um papel mais importante no controlo do peso a longo prazo. ${ }^{15}$ Estes dados sustentam a necessidade e pertinência da realização de intervenções ao nível da alimentação, com reforço junto de populações migrantes, particularmente daqueles com maior risco cardiovascular e avaliação de diferenças de acordo com a origem na resposta à intervenção.

O estudo PRAdA, "Proposta de Alteração da Alimentação em duas populações distintas: hipertensos medicados imigrantes e não-imigrantes dos cuidados de saúde primários da região de Lisboa e Vale do Tejo", consiste na avaliação de uma intervenção ao nível do controlo da PA e da melhoria dos conhecimentos e práticas alimentares. $\mathrm{O}$ presente estudo tem como principal objetivo avaliar o efeito da intervenção no peso dos participantes de ambos os grupos. São objetivos secundários identificar fatores de sucesso na perda de peso em ambos os grupos e avaliar os conhecimentos adquiridos e a adesão às recomendações alimentares no final da intervenção.

\section{MATERIAL E MÉTODOS}

Estudo constituído por uma primeira fase observacional para caracterizar os conhecimentos, hábitos alimentares e os estilos de vida dos participantes e uma segunda fase de intervenção (seis meses) para o controlo da PA e perda de peso através de alterações na alimentação e estilos de vida. Os IOA foram definidos como tendo nascido num dos PALOP (Cabo Verde, São Tomé e Príncipe, Moçambique, Angola e Guiné-Bissau) e tendo origem africana. Os NOP foram definidos como tendo nascido em Portugal e sendo caucasianos. Os participantes foram selecionados aleatoriamente da lista de utentes de quatro unidades de saúde da região de Lisboa selecionadas por conveniência para o estudo. Os critérios de inclusão foram: tomar medicação anti-hipertensiva; idade entre 40 e 85 anos; diagnóstico clínico de HTA; inscrição num dos Centros de Saúde (CS) ou de Unidades de Saúde Familiar (USF) selecionados; contactável por telefone, morada registada no sistema de informação; não estar a ser orientado por um Nutricionista/ Dietista, para perder peso ou controlo da HTA. Os participantes da fase de intervenção foram selecionados a partir da fase observacional tendo que manter os critérios iniciais e ter excesso de peso.

Após o convite por carta, os participantes foram contactados por telefone e foi aplicado um questionário de elegibilidade. Dos participantes elegíveis, os que aceitaram participar no estudo foram convidados a comparecer inicialmente apenas para recolha de dados (fase observacional) e posteriormente na primeira entrevista da fase de intervenção, no respetivo CS/USF, sendo que em ambas as fases foi entregue o consentimento informado escrito.

Este projeto foi autorizado pelas Comissões de Ética da Faculdade de Medicina de Lisboa e da Administração Regional de Saúde de Lisboa e Vale do Tejo e obteve o parecer positivo da Comissão Nacional de Proteção de Dados.

\section{Fase observacional}

$\mathrm{Na}$ fase observacional do estudo foi realizada uma entrevista presencial com a duração aproximada de $30 \mathrm{mi}-$ nutos onde foram recolhidos dados antropométricos (peso, altura, perímetro da cintura), foi calculado o IMC, avaliada a tensão arterial e o perfil de consumo alimentar e de sódio, bem como o consumo de álcool, hábitos tabágicos e atividade física. A variação do peso entre a entrevista observacional e a primeira entrevista da fase de intervenção foram posteriormente comparados com a variação de peso ao longo da fase de intervenção.

\section{Intervenção}

No período dos seis meses foram realizadas três sessões presenciais individuais (0, 3 e 6 meses), com a duração aproximada de uma hora e duas entrevistas intercalares de follow-up telefónicas (1,5 e 4,5 meses). As entrevistas foram conduzidas por profissionais da área de dietética e de nutrição. Na primeira sessão presencial, após recolha de dados para caracterização à entrada no estudo, foram dadas as recomendações gerais de uma alimentação sau- 
Tabela 1 - Recomendações alimentares do estudo PRAdA

\begin{tabular}{ll}
\hline Recomendações & Frequência de consumo \\
\hline$N^{\circ}$ refeições diárias & $\geq 4$ refeições ao longo do dia, incluindo pequeno-almoço ${ }^{16}$ \\
Fruta & $\geq 3$ vezes por dia ${ }^{17}$ \\
Hortícolas & $\geq 3$ vezes por dia ${ }^{17}$ \\
Pescado & peixe fresco ou congelado $\geq 2$ vezes por semana, sendo que uma delas deve ser peixe gordo ${ }^{18}$ \\
Carnes vermelhas & $\leq 2$ vezes por semana ${ }^{19}$ \\
Ovos & $\leq 4$ ovos/semana ${ }^{20}$ \\
Leguminosas & $\geq 2$ vezes/semana \\
Lacticínios magros & $\geq 2$ vezes/dia ${ }^{20}$ \\
\hline
\end{tabular}

dável ${ }^{16-20}$ (Tabela 1) e o caderno do participante (ver descrição no tópico Variáveis).

Foi incentivada a prática de 30 minutos de atividade física moderada na maioria dos dias. ${ }^{20}$ Aos fumadores, foi aconselhado deixar de fumar. ${ }^{21}$ Nas entrevistas de follow-up foi avaliada a adesão e reforçadas as recomendações.

\section{Recolha de dados e variáveis}

A PA foi medida com esfigmomanómetro nas quatro entrevistas presenciais. Foi considerada a média de três medições, efetuadas com cinco minutos de intervalo. Foram considerados hipertensos controlados os participantes com valores sistólicos de $<140 \mathrm{mmHg}$ e diastólicos de $<90$ $\mathrm{mmHg} .{ }^{22} \mathrm{O}$ perímetro da cintura (PC) foi medido no ponto médio entre a última costela flutuante e o rebordo superior da crista-ilíaca. A medição do peso foi feita com uma balança digital e a altura com estadiómetro de acordo com o protocolo das medições antropométricas definido noutros estudos. ${ }^{23}$ Todos os valores foram registados e arredondados às décimas $(0,1 \mathrm{~kg} / \mathrm{cm})$.

$\mathrm{O}$ IMC foi calculado aplicando a fórmula: IMC = Peso (kg) / Altura $^{2}(\mathrm{~m})$ e posteriormente os participantes divididos nas categorias preconizadas pela Organização Mundial da Saúde para os adultos. ${ }^{24}$ Devido à heterogeneidade existente na população idosa ( $\geq 65$ anos) e fenómenos que se traduzem numa alteração do IMC, ${ }^{25,26}$ foi utilizada a classificação do Nutritional Screening Initiative (NSI) dependente do sexo e da idade para os participantes com mais de 65 anos. $^{27}$

Os participantes foram classificados com risco de desenvolvimento de complicações metabólicas com valores de perímetro da cintura, acima de $88 \mathrm{~cm}$ para as mulheres e $102 \mathrm{~cm}$ para os homens. ${ }^{28}$

O perfil de consumo alimentar foi avaliado através da aplicação de um questionário de hábitos alimentares e de estilos de vida com questões sobre: número de refeições; ingestão de pequeno-almoço; hortofrutícolas; leguminosas; peixe; carne; laticínios magros; práticas alimentares relacionadas com o consumo de sal; consumo de álcool; tabaco; prática de atividade física.

O consumo de sódio foi estimado através de um questionário de frequência alimentar que avaliou o consumo de 19 grupos de alimentos ricos em sódio ( $\geq 500$ mg sódio/ $100 \mathrm{~g}$ alimento). O consumo estimado foi dividido por 0,8 , uma vez que estudos anteriores indicaram que $80 \%$ da ingestão de sódio provem de alimentos processados e alimentos consumidos fora de casa. ${ }^{29}$ Esta percentagem foi considerada para estimar a ingestão diária de sódio, de modo a que pudesse ser comparada com o objetivo de consumo de sódio recomendado $(<1500 \mathrm{mg}){ }^{20}$

O consumo excessivo de álcool foi definido como sendo superior a duas bebidas diárias nos homens e uma bebida nas mulheres (> $30 \mathrm{~g} \mathrm{e}>15 \mathrm{~g}$ de etanol, respetivamente). ${ }^{17}$ Os participantes foram definidos como estando em conformidade com a recomendação de atividade física, se praticavam 150 minutos de atividade física moderada a intensa $(>3 \mathrm{METs})^{30}$ por semana e/ ou 60 minutos de atividade física vigorosa (> 6METs) por semana. ${ }^{31,32}$ Andar a pé não foi considerado como atividade física moderada uma vez que os participantes reportaram fazer caminhadas em terreno plano e de baixa intensidade.

A insegurança alimentar foi definida como a disponibilidade limitada ou incerta de alimentos nutricionalmente adequados e seguros, ou a capacidade limitada ou incerta para adquirir alimentos aceitáveis em formas socialmente aceitáveis. ${ }^{33}$ Para avaliar situações de insegurança alimentar do agregado familiar dos participantes foi utilizado o questionário de insegurança alimentar traduzido e adaptado do ERS/USDA. ${ }^{34}$

Foram registadas características sociodemográficas e clínicas, com base num questionário construído para o efeito.

\section{Análise Estatística}

Para determinar as características sociodemográficas da amostra total e por grupos foi feita uma análise descritiva, calculadas as medidas de tendência central e dispersão, e comparadas as duas populações recorrendo aos testes $\chi^{2}$ para variáveis categóricas e t de Student ou Mann-Whitney para variáveis contínuas.

Para avaliar as alterações entre o início e o final do estudo foram utilizados os testes de McNemar para variáveis categóricas e os testes t de Student e Wilcoxon emparelhados. Foi considerado o nível de significância de 5\% para todos os testes. Foi testada a associação entre as diversas 
variáveis e a percentagem de variação de peso recorrendo a modelos de regressão linear simples.

$\mathrm{Na}$ análise de regressão linear múltipla para identificar os fatores associados à perda de peso, foram consideradas as variáveis independentes com nível de significância inferior a $20 \%$ na análise univariada. ${ }^{35}$ Todos os modelos múltiplos foram ajustados para o sexo e a idade. Utilizámos o pacote estatístico Statistical Package for Social Sciences (SPSS), versão 20.0, para Windows.

\section{RESULTADOS}

Dos 186 participantes avaliados na fase observacional e que foram contactados para a fase de intervenção, 15 não aceitaram participar, 19 não eram elegíveis por serem acompanhados por nutricionista/dietista, não estarem a tomar medicação para a HTA, ou por não estarem contactáveis. Foram avaliados na primeira entrevista da fase de intervenção 152 participantes dos quais 30 foram excluídos por não apresentarem excesso de peso. Registou-se a perda em seguimento de 12 participantes, por mudança de residência (incluindo mudança para país de origem), perda de contacto, desistência do estudo e morte (Fig. 1)

Dos 110 participantes que completaram a intervenção, 60 eram IOA ( $71,7 \%$ mulheres) e 50 NOP $(58,0 \%$ mulhe- res). Os IOA participantes estavam em Portugal em média há $24,38 \pm 11,75$ anos. Quanto ao nível educacional $74,8 \%$ concluíram apenas $01^{\circ}$ ou $2^{\circ}$ ciclos de escolaridade (Tabela 2), não existindo diferenças estatisticamente significativas entre os dois grupos. Verificou-se ainda que a maioria era casada ou vivia maritalmente $(65,7 \%)$. Os NOP tinham uma idade média 6 anos superior à dos IOA.

No início do estudo, os valores de pressão arterial diastólica eram, em média, $5 \mathrm{mmHg}$ mais elevados nos IOA $(p<0,05)$. Não foram observadas diferenças estatisticamente significativas entre os dois grupos quanto à pressão arterial sistólica, número de fármacos anti-hipertensivos ou auto reporte de diabetes ou hipercolesterolémia. O IMC médio foi $31,6 \mathrm{~kg} / \mathrm{m}^{2}$ e $59,1 \%$ dos participantes apresentaram obesidade, não se verificando diferenças significativas entre os grupos. Constatou-se que a maioria dos IOA viviam em agregados familiares com situação de insegurança alimentar $(73,3 \%)$ comparativamente a $14,0 \%$ dos NOP $(p<0,01)$.

$\mathrm{Na}$ Tabela 3 são apresentados os resultados da intervenção. Quanto à variação de peso $(0,95 \mathrm{~kg})$ e IMC $(0,35$ $\mathrm{kg} / \mathrm{m}^{2}$ ) ao longo da intervenção, foi significativa para a amostra total, mas, na análise segundo os grupos, a diminuição do peso só foi significativa nos $\operatorname{NOP}(p<0,001)$. Por

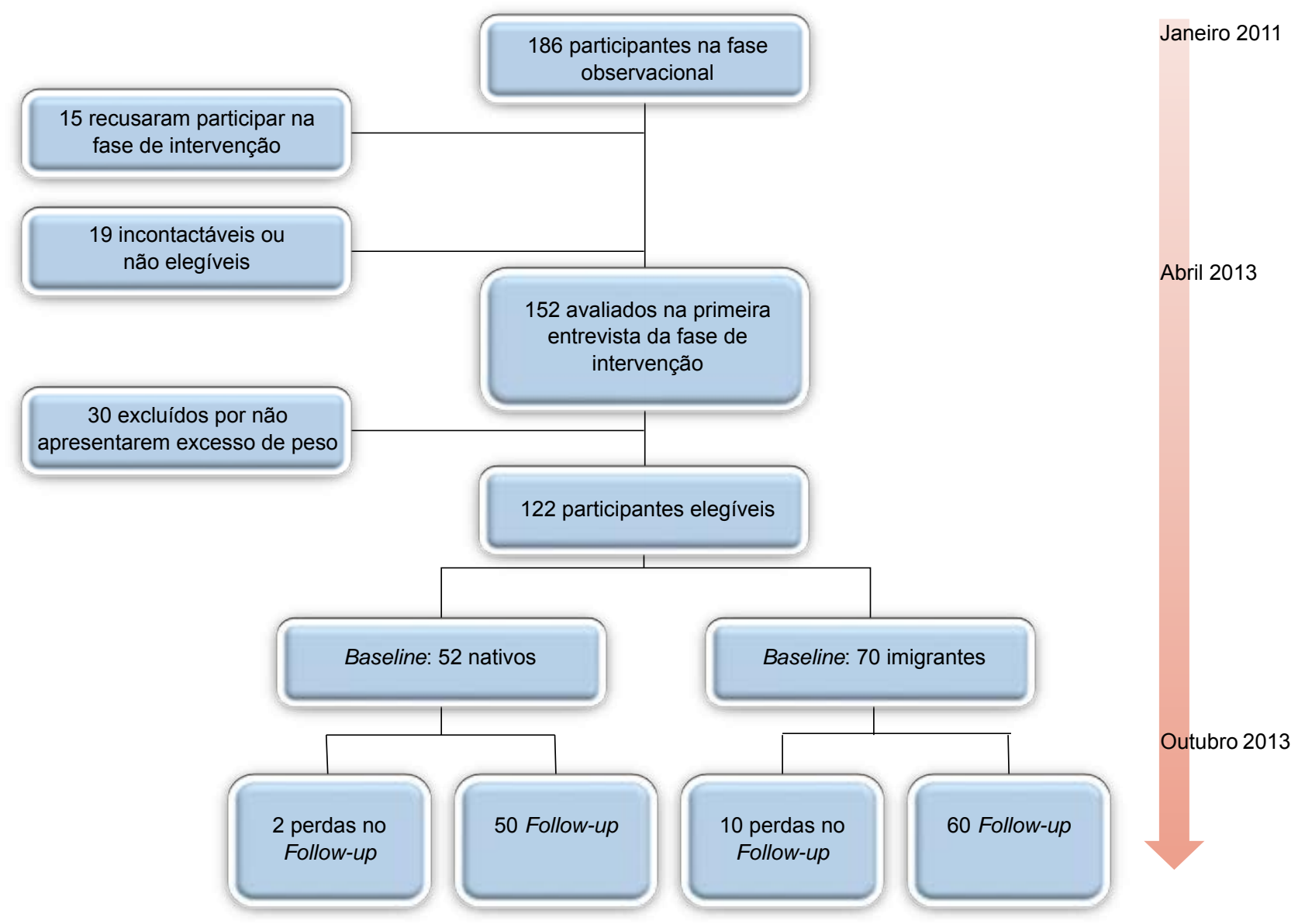

Taxa de adesão: $91 \%$

Figura 1 - Fluxograma 
Tabela 2 - Características socioeconómicas e clínicas dos participantes na amostra total e por origem no início do estudo

\begin{tabular}{|c|c|c|c|c|c|}
\hline & & $\begin{array}{c}\text { Total } \\
(n=110)\end{array}$ & $\begin{array}{l}\text { Nativos } \\
(n=50)\end{array}$ & $\begin{array}{l}\text { Imigrantes } \\
(n=60)\end{array}$ & Valor-p \\
\hline \multicolumn{6}{|c|}{ Características sociodemográficas } \\
\hline \multicolumn{2}{|l|}{ Idade, anos, (média \pm DP) } & $61,3 \pm 10,0$ & $64,6 \pm 8,5$ & $58,6 \pm 10,3$ & $<0,01$ \\
\hline \multirow{2}{*}{ Idade $(\%, n)$} & {$[40-64]$ anos } & $62,7(69)$ & $54,0(27)$ & $70,0(42)$ & \multirow{2}{*}{0,08} \\
\hline & [65-85] anos & $37,3(41)$ & $46,0(21)$ & $30,0(18)$ & \\
\hline Sexo $(\%, n)$ & Homens & $34,5(38)$ & $42,0(21)$ & $28,3(17)$ & 0,13 \\
\hline \multicolumn{2}{|c|}{ Anos de escolaridade, média \pm DP } & $5,2 \pm 3,3$ & $5,5 \pm 3,2$ & $5,0 \pm 3,5$ & 0,63 \\
\hline \multirow{3}{*}{ Nível educacional $(\%, n)$} & $1^{\circ}$ e $2^{\circ}$ ciclo & $74,8(80)$ & $76,0(38)$ & $73,3(42)$ & \multirow{3}{*}{0,89} \\
\hline & $3^{\circ}$ ciclo e secundário & $22,4(24)$ & $22,0(11)$ & $22,8(13)$ & \\
\hline & Ensino superior ou outros & $2,8(3)$ & $2,0(1)$ & $3,5(2)$ & \\
\hline \multirow{3}{*}{ Estado civil $(\%, n)$} & Solteiro & $15,7(17)$ & $4,0(2)$ & $25,9(15)$ & \multirow{3}{*}{0,08} \\
\hline & Casado /união de facto & $65,7(71)$ & $74,0(37)$ & $58,6(34)$ & \\
\hline & Outros & $17,6(19)$ & $22,0(11)$ & $15,5(9)$ & \\
\hline \multicolumn{2}{|c|}{ Insegurança Alimentar $(\%, n)$} & $46,4(51)$ & $14,0(7)$ & $73,3(44)$ & $<0,01$ \\
\hline \multicolumn{6}{|l|}{ Dados clínicos } \\
\hline \multicolumn{2}{|c|}{ Pressão sistólica mmHg (média \pm DP) } & $134,2 \pm 20,6$ & $132,5 \pm 17,2$ & $135,6 \pm 22,7$ & 0,43 \\
\hline \multicolumn{2}{|c|}{ Pressão diastólica mmHg (média \pm DP) } & $82,5 \pm 13,1$ & $79,8 \pm 10,1$ & $84,8 \pm 14,8$ & 0,04 \\
\hline \multicolumn{2}{|c|}{ Pressão arterial controlada $(\%, n)$} & $56,4(62)$ & $62,0(31)$ & $51,7(31)$ & 0,28 \\
\hline \multicolumn{2}{|c|}{ Número de fármacos anti-hipertensivos (média $\pm \mathrm{DP}$ ) } & 1,75 & 1,62 & 1,85 & 0,37 \\
\hline \multicolumn{2}{|l|}{ IMC $\left(\mathrm{kg} / \mathrm{m}^{2}\right)$ (média $\left.\pm \mathrm{DP}\right)$} & $31,6 \pm 3,7$ & $31,4 \pm 3,4$ & $31,8 \pm 4,0$ & 0,54 \\
\hline \multirow{2}{*}{ IMC $\left(\mathrm{kg} / \mathrm{m}^{2}\right)(\%, n)$} & Pré Obesidade & $40,9(45)$ & $42(21)$ & $40(24)$ & \multirow{2}{*}{0,83} \\
\hline & Obesidade & $59,1(65)$ & $58(29)$ & $60(36)$ & \\
\hline \multicolumn{2}{|c|}{ Perímetro da cintura $(\mathrm{cm})$ (média $\pm \mathrm{DP})$} & $102,2 \pm 9,3$ & $102,7 \pm 10,5$ & $101,7 \pm 8,3$ & 0,55 \\
\hline \multicolumn{2}{|c|}{ Risco cardiovascular (baseado no PC) $(\%, n)$} & $82,6(90)$ & $83,1(49)$ & $82,0(41)$ & 0,54 \\
\hline \multicolumn{2}{|l|}{ Diabetes $(\%, n)$} & $32,7(36)$ & $26,0(13)$ & $38,3(23)$ & 0,23 \\
\hline \multicolumn{2}{|l|}{ Hipercolesterolémia $(\%, n)$} & $66,4(73)$ & $58,0(29)$ & $48,3(29)$ & 0,29 \\
\hline
\end{tabular}

DP - desvio-padrão; IMC - Índice de Massa Corporal; PC - perímetro da cintura

outro lado, a redução do perímetro da cintura foi estatisticamente significativa na amostra total e nos dois grupos.

Quanto ao consumo excessivo de álcool, tabagismo e atividade física não se verificaram diferenças significativas entre o início e final do estudo.

Constatou-se (Tabela 3) que apenas $17,3 \%$ dos participantes $(n=19)$ cumpriam a recomendação de atividade física no início do estudo, com predomínio dos NOP $(28,0 \%$ vs $8,3 \%, p=0,01)$.

No início do estudo, a amostra apresentava uma média de consumo de álcool de 10,43 $\pm 21,81 \mathrm{gr} /$ dia e que foi reduzido em 3,64 gr ao longo do estudo. O consumo de álcool nos NOP no início do estudo era em média 6,93 gr superior ao dos IOA, diferença que se manteve semelhante no final da intervenção.

Relativamente ao conhecimento e seguimento das recomendações alimentares verificou-se um aumento estatisticamente significativo no número médio de recomenda- ções conhecidas e cumpridas, quer na amostra total, quer em ambos os grupos.

Porém, se quanto ao conhecimento das recomendações alimentares não havia diferença significativa entre os grupos, o grupo dos IOA distinguiu-se pelo seu menor cumprimento $(p<0,01)$ quer no início quer no final da intervenção.

A evolução da proporção de participantes que conheciam e cumpriam cada uma das recomendações individuais é apresentada na Fig. 2.

À exceção da recomendação relativa ao consumo de ovos, que todos os participantes cumpriam no início do estudo, verificou-se, no final da intervenção, um aumento da proporção de indivíduos que conheciam e cumpriam todas as recomendações alimentares.

Para avaliar a eficácia da intervenção, foi comparada a percentagem de peso perdido pelos mesmos participantes entre a entrevista observacional do estudo (Janeiro de 2011) e o início da intervenção (Abril de 2012) com a per- 
Tabela 3 - Comparação de conhecimentos, comportamentos alimentares, tabagismo e prática da atividade física entre os grupos durante o período de follow-up

\begin{tabular}{|c|c|c|c|c|c|}
\hline & & $\begin{array}{l}\text { Amostra Total } \\
\quad(n=110)\end{array}$ & $\begin{array}{l}\text { Nativos } \\
(n=50)\end{array}$ & $\begin{array}{l}\text { Imigrantes } \\
(n=60)\end{array}$ & Valor-p \\
\hline \% Peso Perdido (média \pm DP) & & $1,09 \pm 3,2$ & $1,4 \pm 2,7$ & $0,8 \pm 3,6$ & 0,32 \\
\hline \multirow{2}{*}{ Peso, kg (média \pm DP) } & Baseline & $80,84 \pm 11,88$ & $78,81 \pm 11,75$ & $82,52 \pm 11,82$ & 0,10 \\
\hline & 6 meses & $79,89 \pm 11,54$ & $77,55 \pm 10,65$ & $81,84 \pm 11,98$ & 0,52 \\
\hline valor-p - Comparação entre baseline e follow-up & & $<0,01$ & $<0,01$ & 0,09 & \\
\hline \multirow{2}{*}{ IMC, $\mathrm{kg} / \mathrm{m}^{2}$ (média $\pm \mathrm{DP}$ ) } & Baseline & $31,59 \pm 3,69$ & $31,35 \pm 3,36$ & $31,79 \pm 3,95$ & 0,62 \\
\hline & 6 meses & $31,24 \pm 3,73$ & $30,88 \pm 3,17$ & $31,54 \pm 4,14$ & 0,35 \\
\hline valor-p - Comparação entre baseline e follow-up & & 0,01 & $<0,01$ & 0,11 & \\
\hline \multirow{2}{*}{ Perímetro da Cintura, cm (média \pm DP) } & Baseline & $102,19 \pm 9,33$ & $102,77 \pm 0,48$ & $101,69 \pm 8,29$ & 0,55 \\
\hline & 6 meses & $100,61 \pm 9,44$ & $101,08 \pm 9,33$ & $100,22 \pm 9,57$ & 0,64 \\
\hline valor-p - Comparação entre baseline e follow-up & & $<0,01$ & 0,01 & 0,02 & \\
\hline \multirow{2}{*}{ Consumo excessivo de álcool $(\%, n)$} & Baseline & $17,3(19)$ & $26,0(13)$ & $10,0(6)$ & 0,27 \\
\hline & 6 meses & $11,8(13)$ & $22,0(11)$ & $3,3(2)$ & 0,03 \\
\hline valor-p - Comparação entre baseline e follow-up & & 0,34 & 0,62 & 0,12 & \\
\hline \multirow{2}{*}{ Gramas de álcool/dia (média \pm DP) } & Baseline & $10,43 \pm 21,81$ & $14,21 \pm 23,82$ & $7,28 \pm 19,61$ & 0,06 \\
\hline & 6 meses & $6,79 \pm 13,52$ & $10,47 \pm 16,55$ & $3,71 \pm 9,43$ & 0,03 \\
\hline valor-p - Comparação entre baseline e follow-up & & 0,01 & 0,01 & 0,01 & \\
\hline \multirow{2}{*}{ Fumador $(\%, n)$} & Baseline & $9,1(10)$ & $6,0(3)$ & $11,7(7)$ & 0,30 \\
\hline & 6 meses & $7,3(8)$ & $4,0(2)$ & $10,0(6)$ & 0,28 \\
\hline valor-p - Comparação entre baseline e follow-up & & 0,50 & 1,00 & 1,00 & \\
\hline \multirow{2}{*}{ Cumpre recomendação atividade física $(\%, n)$} & Baseline & $19(17,3 \%)$ & $14(28,0 \%)$ & $5(8,3 \%)$ & 0,01 \\
\hline & 6 meses & $17(15,5 \%)$ & $11(22,0 \%)$ & $6(10,0 \%)$ & 0,08 \\
\hline valor-p - Comparação entre baseline e follow-up & & 0,79 & 0,45 & 1,00 & \\
\hline \multirow{2}{*}{ Número de recomendações conhecidas (média \pm DP) } & Baseline & $4,42 \pm 1,85$ & $4,80 \pm 1.67$ & $4,12 \pm 1,96$ & 0,07 \\
\hline & 6 meses & $7,19 \pm 1,13$ & $7,34 \pm 1,02$ & $7,08 \pm 1,21$ & 0,23 \\
\hline valor-p - Comparação entre baseline e follow-up & & $<0,01$ & $<0,01$ & $<0.01$ & \\
\hline \multirow{2}{*}{ Número de recomendações cumpridas (média \pm DP) } & Baseline & $4,01 \pm 1,64$ & $4,27 \pm 1,48$ & $3,76 \pm 1,59$ & 0,05 \\
\hline & 6 meses & $4,93 \pm 1,29$ & $5,42 \pm 1,09$ & $4,39 \pm 1,41$ & $<0,01$ \\
\hline valor-p - Comparação entre baseline e follow-up & & 0,01 & 0,01 & 0,01 & \\
\hline
\end{tabular}

centagem de peso perdido na fase de intervenção. O valor médio de percentagem de peso perdido foi $0,04 \%$ por mês na fase de observação e $0,17 \%$ por mês nos seis meses do estudo de intervenção, ou seja, um efeito suplementar de perda de peso de $0,13 \%$ com a intervenção $(p=0,04)$.

\section{Fatores associados à perda de peso nos dois grupos}

$\mathrm{Na}$ Tabela 4 apresenta-se a análise bivariada entre as características dos participantes e a \% de variação de peso, nas duas populações. Verificou-se que, tanto na amostra total como por grupo, o sexo masculino foi o que mais reduziu o peso. Os indivíduos com HTA inicial não controlada de ambos os grupos apresentam tendência para uma maior perda de peso. Nos NOP verificou-se uma relação direta entre o IMC, à entrada no estudo, e a redução do peso durante a intervenção. Ainda em relação a variáveis clínicas, nos IOA verificou-se uma associação significativa entre ter dislipidémia e maior perda de peso. Quanto ao conhecimento e cumprimento das recomendações alimentares não se encontraram associações significativas com a perda de peso, exceto em relação ao consumo de pelo menos três peças de fruta por dia e pelo menos duas porções de lacticínios magros e conhecimento das recomendações de hortícolas no grupo dos nativos no início do estudo. Quanto ao cumprimento e conhecimento das recomendações alimentares verificou-se no follow-up seis meses uma associação entre o consumo de laticínios magros $\geq 2 \mathrm{x} /$ dia e a perda de peso.

\section{Fatores independentemente associados à perda de peso nos dois grupos}

$\mathrm{Na}$ Tabela 5 apresentam-se os resultados dos modelos de regressão linear múltipla para a amostra total e para cada uma das populações, NOP e IOA, respetivamente. $\mathrm{Na}$ população total, o sexo mostrou ser um fator preditor da perda de peso, com os homens a apresentarem uma percentagem de peso perdido estatisticamente superior $(p=0,01)$. O consumo de laticínios magros $\geq 2 \mathrm{vezes} / \mathrm{dia}$ 

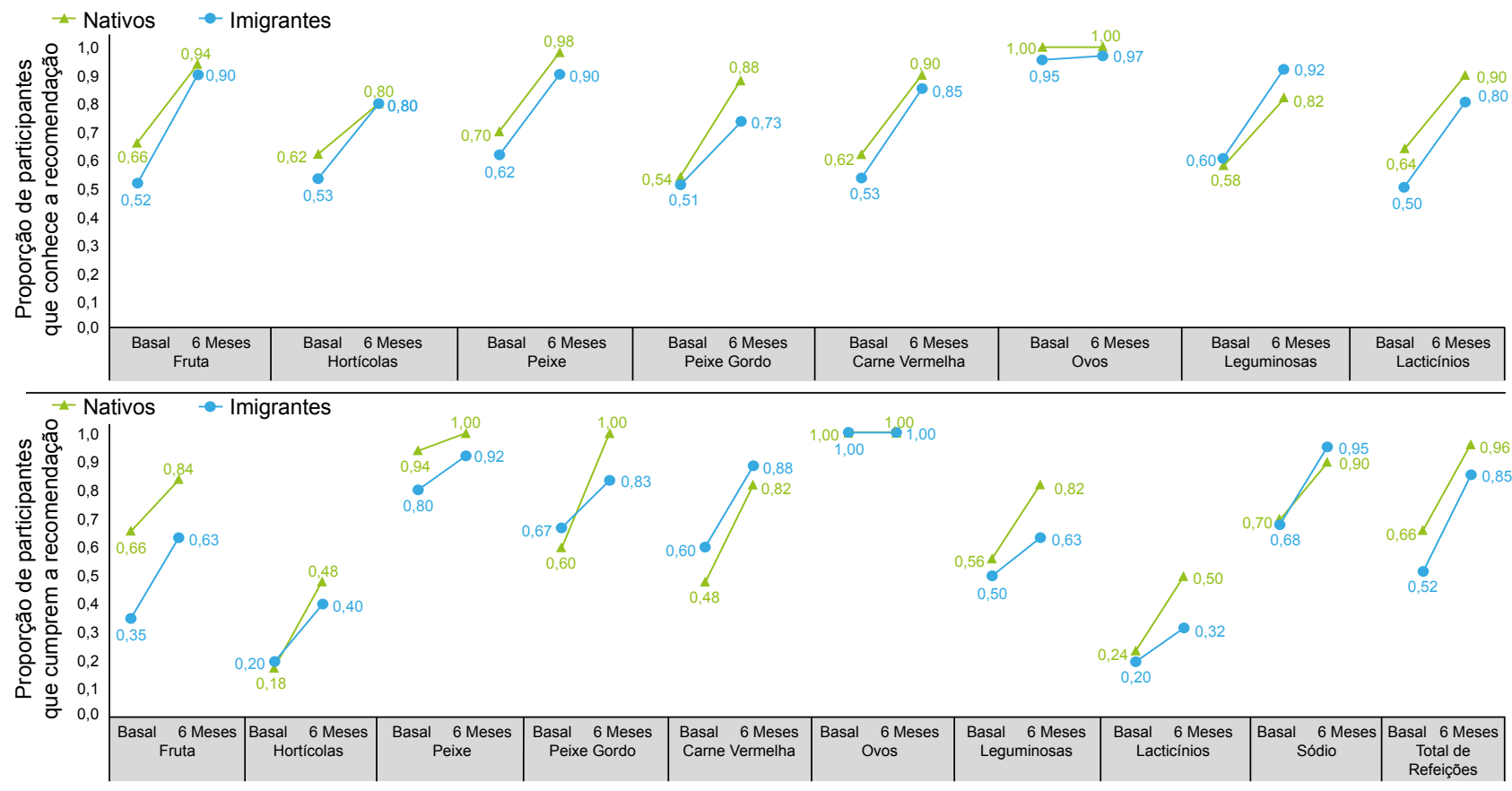

Figura 2 - Evolução da proporção de participantes que conhecem e que cumprem cada uma das recomendações

no momento inicial do estudo mostrou estar associado com uma maior percentagem de perda de peso na amostra total $(p=0,01)$.

No grupo dos NOP, o consumo de pelo menos três porções de fruta por dia mostrou-se associado à perda de peso. No grupo dos IOA, verificaram-se apenas tendências de associação entre a dislipidémia e o consumo de leguminosas com maior percentagem de perda de peso.

\section{DISCUSSÃO}

A intervenção comportamental do estudo PRAdA demonstrou ser eficaz na promoção da redução de peso em hipertensos medicados, com excesso de peso, seguidos em cuidados de saúde primários da Região de Lisboa. Comparando com a fase observacional, a percentagem de peso perdido foi, por mês, $0,13 \%$ superior durante a intervenção. Contudo, esta intervenção parece ser mais eficaz nos NOP (em comparação com os IOA) e nos homens (em comparação com as mulheres). A maior redução do peso nos NOP que nos IOA ( $1,4 \%$ vs $0,8 \%)$ confirma a tendência observada noutros estudos. ${ }^{7,8,36}$ Todavia, apesar da proporção entre as diferenças de perda de peso ser semelhante, o valor médio de perda de peso em seis meses de intervenção $(1,26 \mathrm{~kg}$ nos NOP e $0,68 \mathrm{~kg}$ nos IOA) não pode ser diretamente comparado com estes estudos, com perdas entre $2,3 \mathrm{~kg}$ e $5,7 \mathrm{~kg}$ nos negros e 4,5 kg e 8,0 kg nos caucasianos na medida em que as metodologias de intervenção são distintas. $5,7,8,37$

A maioria destas intervenções foi feita em voluntários, incluíram um maior número de sessões, entre 16 a 30, e centraram-se em três fatores: motivacionais, alimentação e estilos de vida. Os resultados obtidos são similares a outros estudos, que incluíram minorias étnicas, apenas com um componente de intervenção (alimentação ou exercício físi- $\mathrm{co})^{37}$ e que decorreram em cuidados de saúde primários. ${ }^{38}$ Outros fatores que poderão contribuir para a menor perda de peso face a resultados internacionais poderão ser o facto de a intervenção ter sido centrada na dieta $\mathrm{DASH}^{20}$ com controlo do consumo de sal, aumento da ingestão de hortofrutícolas, lacticínios magros, peixe e controlo da ingestão de carnes vermelhas e não na restrição calórica.

Por outro lado, outro indicador da eficácia da intervenção foi o aumento dos conhecimentos e o maior cumprimento das recomendações alimentares, em ambos os grupos. No entanto, apesar dos conhecimentos iniciais sobre as recomendações alimentares e a aquisição de conhecimentos ser semelhante, entre NOP e IOA, verificou-se um menor cumprimento das recomendações por parte dos IOA. Isto sugere uma menor capacidade de adaptação às recomendações, provavelmente associados a fatores culturais e processos de aculturação. ${ }^{37} \mathrm{Com}$ efeito, a imigração tradicional Portuguesa é oriunda dos PALOP e o seu fluxo iniciou em meados da década de setenta. Apesar do elevado grau de aculturação, as práticas alimentares mantêm em larga medida as raízes culturais dos países de origem. Outro fator que importa ainda referir é a percentagem de indivíduos em situação de insegurança alimentar, significativamente superior no grupo dos IOA em comparação com os NOP. Alguns estudos de revisão têm demonstrado associação entre Insegurança Alimentar e obesidade, maioritariamente nas mulheres devido não há falta de conhecimentos mas sim devido a falta de acesso a opções mais saudáveis. ${ }^{39}$ Alguns autores sugerem que os indivíduos de raça negra, mesmo aqueles suficientemente motivados para a mudança, parecem ter uma menor perda de peso inicial por necessitarem de mais tempo para identificar estratégias viáveis e adaptá-las ao seu contexto pessoal. ${ }^{8,40}$ 


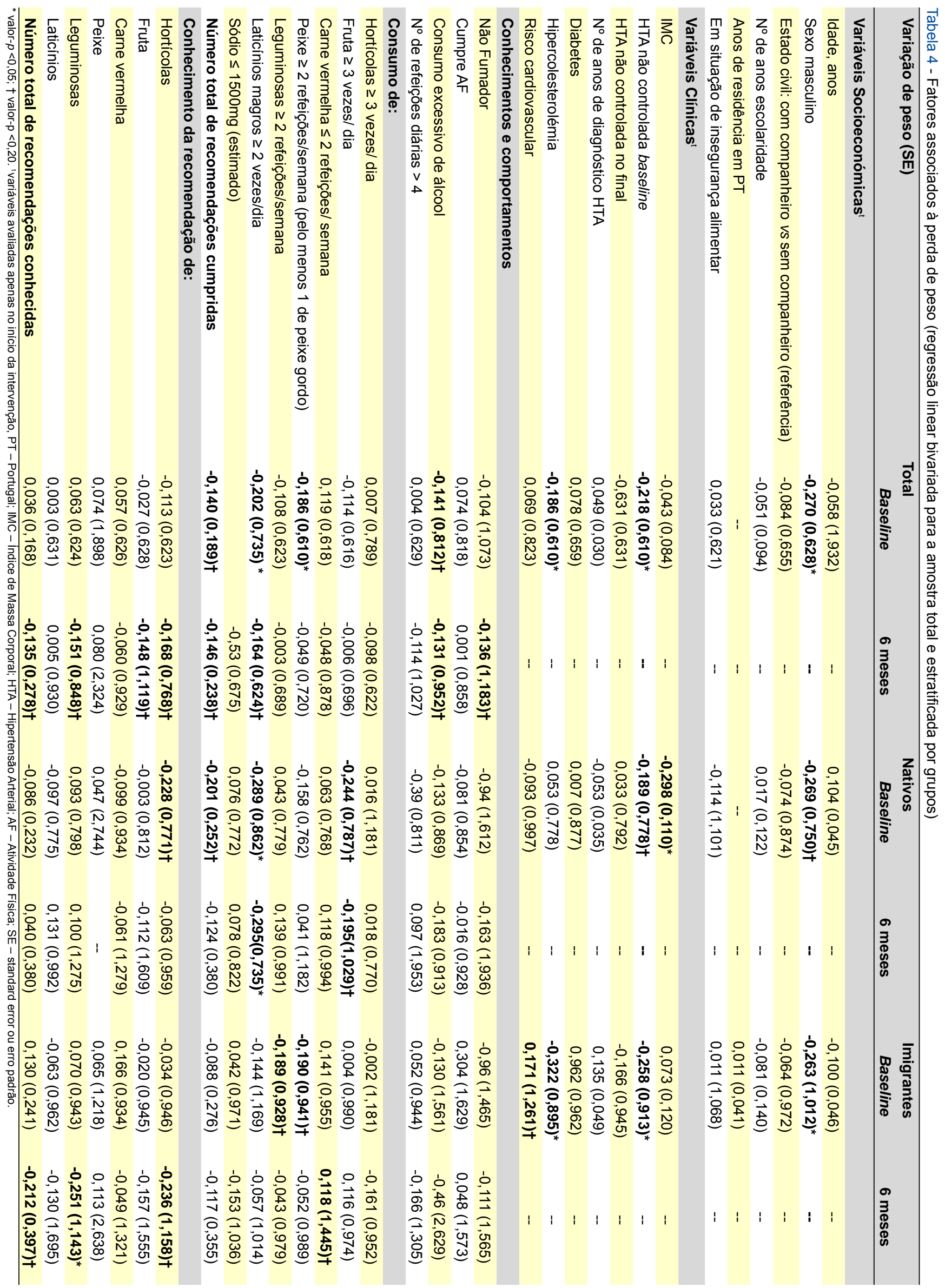


Neste estudo, a perda de peso, com o ajustamento para a idade, foi maior no sexo masculino, tanto na amostra total como em ambos os grupos. Este resultado é consistente com outras investigações, admitindo-se que estas diferenças entre os sexos tenham uma base biológica e associada à resposta ao exercício físico. ${ }^{41}$ Verificou-se ainda a associação de fatores de risco cardiovascular - HTA não controlada, IMC mais elevado no grupo dos NOP, hipercolesterolémia no grupo dos NOP - com maiores perdas de peso ao longo da intervenção. Estes dados são sugestivos de que um maior risco cardiovascular é um fator de motivação para adesão às recomendações traduzindo-se em maior perda de peso.

No grupo de NOP o consumo de pelo menos três peças de fruta por dia e duas porções de lacticínios magros está também associado a uma maior perda de peso. Já no grupo de IOA verificam-se apenas tendências de associação com conhecimentos alimentares.

Uma das limitações do estudo está relacionada com a dimensão da amostra para realizar modelos de regressão múltipla. Com um tamanho amostral de 50 para o grupo dos NOP e 60 para o dos IOA, apenas é possível obter um poder estatístico de 0,70 e uma diferença (effect size) de 0,35 . Com um tamanho amostral de 120 , seria possível obter um poder estatístico de 0,80 e uma diferença de 0,15, valores comummente utilizados neste tipo de análises. ${ }^{42}$ Também a inclusão de várias unidades de saúde de uma região, apesar da subsequente seleção aleatória de participantes, não garante a generabilidade dos resultados para o restante território nacional.

Outra limitação da intervenção proposta é a sua curta duração, menor que a maioria dos estudos nesta área. ${ }^{37}$ Esta opção foi justificada pelo contexto de aplicação da intervenção ao nível dos cuidados de saúde primários mas

Tabela 5 - Fatores associados à perda de peso - regressão linear múltipla para a amostra total e estratificada por grupos

\begin{tabular}{|c|c|c|c|c|c|c|}
\hline & \multicolumn{2}{|c|}{ Amostra total* } & \multicolumn{2}{|c|}{ Nativos } & \multicolumn{2}{|c|}{ Imigrantes } \\
\hline & Beta $^{a}$ & valor $p$ & $\beta^{\mathrm{a}}$ & Valor-p & $\beta^{\mathrm{a}}$ & Valor-p \\
\hline Idade & 0,031 & 0,760 & 0,178 & 0,179 & $-0,016$ & 0,919 \\
\hline Sexo Masculino & $-0,281$ & 0,012 & $-0,192$ & 0,147 & $-0,226$ & 0,173 \\
\hline Hipertensão não controlada no início da intervenção & $-0,131$ & 0,172 & $-0,128$ & 0,338 & $-0,115$ & 0,410 \\
\hline Índice Massa Corporal & - & - & $-0,282$ & 0,032 & & \\
\hline Risco cardiovascular (perímetro da cintura) & & & & & 0,100 & 0,523 \\
\hline Não fumador aos 6 meses & $-0,051$ & 0,585 & - & - & & \\
\hline Hipercolesterolémia & $-0,146$ & 0,138 & - & - & $-0,271$ & 0,084 \\
\hline Consumo excessivo de álcool no baseline & 0,036 & 0,730 & - & - & & \\
\hline $\begin{array}{l}\text { Consumo de peixe } \geq 2 \text { refeições/semana } \\
\text { (pelo menos } 1 \text { é refeição de peixe gordo) no baseline }\end{array}$ & $-0,111$ & 0,248 & - & - & $-0,058$ & 0,675 \\
\hline Consumo de fruta $\geq 3$ vezes/dia no baseline & - & - & $-0,276$ & 0,037 & & \\
\hline Consumo de lacticínios magros $\geq 2$ vezes/dia no baseline & $-0,242$ & 0,011 & & & & \\
\hline Consumo de leguminosas $\geq 2$ refeições/semana no baseline & & & & & 0,018 & 0,907 \\
\hline Número de refeições diárias superior a 4 aos 6 meses & & & & & $-0,172$ & 0,250 \\
\hline Consumo de carne vermelha $\leq 2$ refeições/semana aos 6 meses & & & & & 0,021 & 0,874 \\
\hline Consumo de lacticínios magros $\geq 2$ vezes/dia aos 6 meses & - & - & $-0,240$ & 0,064 & & \\
\hline Conhecimento da recomendação de fruta aos 6 meses & $-0,141$ & 0,136 & & & & \\
\hline Conhecimento da recomendação de leguminosas aos 6 meses & $-0,062$ & 0,509 & & & $-0,216$ & 0,124 \\
\hline Conhecimento da recomendação de hortícolas aos 6 meses & $-0,063$ & 0,521 & $-0,145$ & 0,271 & $-0,132$ & 0,339 \\
\hline R2 ajustado; Erro padrão & 0,15 & 2,995 & 0,241 & 2,344 & 0,18 & 3,325 \\
\hline
\end{tabular}

* Modelo para a amostra total ajustado para a origem. Para cada um dos modelos foram selecionadas apenas as variáveis independentes com nível de significância inferior a $20 \%$ na análise univariada. 
pode ter implicação ao nível do sucesso da intervenção. No futuro, seria preponderante reavaliar os participantes de modo a avaliar a eficácia da intervenção a longo prazo. Embora conscientes de que avaliações em diferentes épocas do ano podem estar sujeitas a alterações de peso sazonais, estudos indicam que estas flutuações são ligeiras e que os picos e quedas não são coincidentes com os momentos de avaliação do estudo PRAdA. ${ }^{43}$ Pelo que com o objetivo de minimizar a limitação de ausência de grupo controlo foi comparada a variação de peso entre a entrevista observacional e o início da fase de intervenção do estudo.

Um dos pontos fortes da intervenção é demonstrar a fácil implementação nos cuidados de saúde primários, dado o baixo número de sessões presenciais e de técnicos de saúde necessários. A exequibilidade do estudo é uma garantia de que será possível aplicar esta metodologia a nível nacional em grupos de risco cardiovascular inscritos nos cuidados de saúde primários.

No entanto, os diferentes resultados da intervenção de acordo com o sexo e origem podem indicar caminhos para uma melhor intervenção clínica. A maior dificuldade de adesão às recomendações verificada no grupo dos IOA e não a menor aquisição de conhecimentos deverá ser fator considerado no delineamento das estratégias de intervenção. As estratégias adotadas devem ter em atenção diferenças de âmbito cultural e motivacional das comunidades envolvidas.

É ainda de salientar que apesar da perda de peso ter sido pequena em comparação com a obtida com intervenções intensivas, segundo alguns autores as alterações na alimentação em indivíduos com risco cardiovascular, mesmo não se traduzindo em alterações no peso, melhoram o perfil de risco cardiovascular. ${ }^{44}$ Mesmo perdas ligeiras conduzem a redução nos valores da PA (aproximadamente 1 $\mathrm{mm} \mathrm{Hg}$ por kg de peso perdido), em particular os indivíduos sob terapêutica Anti-hipertensiva. ${ }^{45}$

\section{CONCLUSÃO}

Os resultados da intervenção foram bem-sucedidos no que diz respeito à promoção de perda de peso, especialmente no grupo dos NOP. As diferenças entre grupos de origem relativamente à perda de peso foram as esperadas, ou seja, os IOA perderam menos peso que os NOP.

A intervenção educacional aumentou os conhecimentos e adesão às recomendações alimentares em ambos os grupos, realçando a pertinência da educação alimentar e intervenção ao nível dos estilos de vida nos cuidados de saúde primários, nomeadamente promoção do consumo de hortofrutícolas e peixe e redução do consumo de sal e carnes vermelhas (Tabela 1).

$\mathrm{Na}$ amostra total, os fatores de sucesso associados à perda de peso foram o sexo masculino e o consumo de mais do que duas porções de produtos lácteos com baixo teor de gordura por dia, independente da idade e origem. Nos nativos, o IMC superior e o consumo de pelo menos três porções de fruta, no início da intervenção, também se relacionaram com uma maior perda de peso, independentemente do sexo e idade.

De acordo com os resultados, futuras intervenções deverão não só enfatizar os fatores de sucesso na perda de peso mas também apresentar maior duração e avaliar com maior rigor a ingestão de água e sódio. De modo a facilitar a adesão à dietoterapia e estilos de vida saudáveis deverão ser incluídas técnicas motivacionais e uma maior componente de envolvimento cultural diminuindo as disparidades em saúde. ${ }^{11}$

\section{AGRADECIMENTOS}

Os autores gostariam de agradecer a todos os participantes do estudo, às Unidades de Saúde dos Agrupamentos de Centros de Saúde de Loures (CS Sacavém e USF São João da Talha) e Algueirão - Rio de Mouro (CS Algueirão e USF Natividade), e aos membros do projeto Ana Pinto, Beatriz Goulão e Isabel Cardoso.

\section{CONFLITOS DE INTERESSE}

Os autores declaram não ter conflitos de interesse relativamente ao presente artigo.

\section{FONTES DE FINANCIAMENTO}

O estudo PRAdA foi financiado pela Direção-Geral da Saúde (DGS) e pela Fundação AstraZeneca. Parte do presente trabalho foi apresentada nas seguintes conferências: "Efeito na perda de peso de uma intervenção alimentar e de estilos de vida em hipertensos medicados nativos e imigrantes africanos seguidos nos cuidados de saúde primários da região de Lisboa e Vale do Tejo (Estudo PRAdA)", comunicação oral no XII Congresso de Nutrição e Alimentação, 16 e 17 de Maio 2013, Centro de Congressos de Lisboa; "Effect on weight loss of food and lifestyles intervention in treated hypertensive natives and African immigrants in Lisbon (PRAdA Study)", poster presentation, EUROEPI 2013, 11-14 August, 2013, Aarhus - Denmark.

tárias e em praticantes de exercício físico, na cidade do Porto. Acta Med Port. 2010;23:153-8

4. Pereira S. Abordagem do risco cardiovascular no doente hipertenso. Acta Med Port. 2010;23:223-6.

5. Kumanyika, S. Ethnic minorities and weight control research priorities: where are we now and where do we need to be? Prev Med. 2008;47:583-6.

6. Smith GD, Chaturvedi N, Harding S, Nazroo J. Williams R. Ethnic inequalities in health: A review of UK epidemiological evidence. Crit Public 
Health. 2000;10:375-408

7. Kumanyika SK, Espeland MA, Bahnson JL, Inferior JB, JB Charleston, Folmar S, et al. Ethnic comparison of weight loss in the Trial of Nonpharmacologic Interventions in the Elderly. Obes Res. 2002;10:96-106.

8. West DS, Prewitt ET, Bursac Z, Felix HC. Weight loss of black, white, and Hispanic men and women in the Diabetes Prevention Program. Obesity. 2008;16:1413-20.

9. Fitzgibbon ML, Tussing-Humphreys LM, Porter JS, Martin IK, OdomsYoung A, Sharp LK. Weight loss and African-American women: a systematic review of the behavioural weight loss intervention literature. Obesity Rev. 2012;13:193-213.

10. Serviço Estrangeiros e Fronteiras. Relatório de Imigração, Fronteiras e Asilo 2011. Lisboa, 2012.

11. Instituto Nacional de Estatística. Inquérito Nacional de Saúde 2005/2006. Lisboa, 2009.

12. Estrela P. A saúde dos imigrantes em Portugal. Rev Port Clin Geral. 2009;45-55.

13. Reijneveld SA. Reported health, lifestyles, and use of health care of first generation immigrants in The Netherlands: do socioeconomic factors explain their adverse position? J Epidemiol Community Health. 1998;52:298-304

14. World Health Organization. International Migration, Health Acknowledgements. Geneve: WHO; 2003.

15. Teixeira PJ, Going SB, Houtkooper LB, Cussler EC, Metcalfe LL, Blew $\mathrm{RM}$, et al. Exercise motivation, eating, and body image variables as predictors of weight control. Med Sci Sports Exerc. 2006;38:179-88.

16. ADA position of the American Dietetic Association: weight management. J Am Diet Assoc. 2009;109:330-46.

17. Rodrigues SS, Franchini B, Graça P, de Almeida MD. A new food guide for the Portuguese population: development and technical considerations. J Nutr Educ Behav. 2006;38:189-95.

18. Scientific Advisory Comittee on Nutrition. Advice on fish consumption: benefits \& risks 2004. London: TSO (The Stationery Office); 2004.

19. Pan A, Sun Q, Bernstein AM, Schulze MB, Manson JE, Stampfer MJ, et al. Red meat consumption and mortality: results from 2 prospective cohort studies. Arch Intern Med. 2012;172:555-63.

20. NIH. Lowering your blood pressure with DASH. NIH Publication. 2006; No. 06-4082.

21. National Institute for Health and Clinical Excellence Hypertension: Clinical management of primary hypertension in adults. NICE clinical guideline 127. 2011.

22. Mancia G, De Backer G, Dominiczak A, Cifkova R, Fagard R, Germano G, et al. 2007 ESH-ESC Practice Guidelines for the Management of Arterial Hypertension: ESH-ESC Task Force on the Management of Arterial Hypertension. J Hypertens. 2007;25:1751-62.

23. National Diet and Nutrition Survey Interviewer Measurements. 2007;111.

24. World Health Organization. WHO - Fact Sheet No311. 2011. [Consultado 2013 Jul 08]. Disponível em: http://www.who.int/mediacentre/factsheets/fs311/en/.

25. Green SM, Watson R. Nutritional screening and assessment tools for older adults: literature review. J Advanc Nurs. 2006;54:477-90.

26. Heiat A, Vaccarino V., Krumholz HM. An evidence-based assessment of federal guidelines for overweight and obesity as they apply to elderly persons. Arch Intern Med. 2001:161:1194-203.

27. Blackburn GL, Dwyer JT, Wellman NS, eds in collaboration with American Academy of Family Physicians, the American Dietetic Association, National Council on the Aging. Nutrition interventions manual for professionals caring for older Americans. Washington, DC: The Nutrition Screening Initiative; 1992.

28. Direcção-Geral da Saúde. Programa Nacional de Combate à Obesidade. Lisboa: DGS; 2005.

29. He FJ, MacGregor GA. Reducing population salt intake worldwide: from evidence to implementation. Progr Cardiovasc Dis. 2010;52:363-82.

30. World Health Organization. Global Recommendations on Physical Activity for Health. Geneve: WHO; 2010.

31. Compêndio de actividades físicas. Como calcular o dispêndio energético de actividade física em adultos. Lisboa. FMH Edições; 2004

32. Mendes R, Sousa N; Barata JLT. Actividade física e saúde pública recomendações para a prescrição de exercício. Acta Med Port 2011;24:1025-30.

33. Core indicators of nutritional state for difficult-to-sample populations. $J$ Nutr. 1990;120:1557S-600.

34. USDA/ERS, U. S. D. of A.-E. R. S. U.S. Household Food Security Survei Module: Six Short Form. Philadelphia: USDA/ERS; 2008.

35. Hosmer C, Lemeshow S. Applied logistic regression. New York: Wiley 2000.

36. Hollis JF, Gullion CM, Stevens VJ, Brantley PJ, Appel LJ, Ard JD, et al. Weight loss during the intensive intervention phase of the weight-loss maintenance trial. Am J Prev Med. 2008;35:118-26.

37. Seo DC, Sa J.A meta-analysis of psycho-behavioral obesity interventions among US multiethnic and minority adults. Prev Med. 2008;47:573-82.

38. Logue EE, Jarjoura DG, Sutton KS, Smucker WD, Baughman KR, Capers CF. Longitudinal relationship between elapsed time in the action stages of change and weight loss. Obes Res. 2004;12:1499-508.

39. Dinour LM, Bergen D, Yeh MC. The food insecurity-obesity paradox: a review of the literature and the role food stamps may play. J Am Diet Assoc. 2007;107:1952-61.

40. Epstein DE, Sherwood A, Smith PJ, Craighead L, Caccia C, Lin PH, et al. Determinants and consequences of adherence to the dietary approaches to stop hypertension diet in African-American and white adults with high blood pressure: results from the ENCORE trial. J Acad Nutr Diet. 2012;112:1763-73.

41. Dunn CL, Hannan PJ, Jeffery RW, Sherwood NE, Pronk NP, Boyle R. The comparative and cumulative effects of a dietary restriction and exercise on weight loss. Int J Obes. 2006;30:112-21.

42. Soper DS. A-priori sample size calculator for multiple regression. (2013). [Consultado 2013 Jul 10]. Disponível em: http://www.danielsoper.com/ statcalc.

43. Ma Y, Olendzki BC, Li W, Hafner AR, Chiriboga D, Hebert JR, et al. Seasonal variation in food intake, physical activity, and body weight in a predominantly overweight population. Eur J Clin Nutr. 2006;60:519-28.

44. Gaesser GA, Angadi SS, Sawyer BJ. Exercise and diet, independent of weight loss, improve cardiometabolic risk profile in overweight and obese individuals. Phys Sportsmed. 2011;39:87-97.

45. Neter JE, Stam BE, Kok FJ, Grobbee DE, Geleijnse JM. Influence of weight reduction on blood pressure: a meta-analysis of randomized controlled trials. Hypertension. 2003;42:878-84. 
Catarina PEIXOTO, Gisela CARRILHO, Violeta ALARCÃO, Filipa GUERRA, Rui SIMÕES, Milene FERNANDES, Paulo NICOLA, Sofia GUIOMAR, Paulo NOGUEIRA, Evangelista ROCHA

Intervenção PRAdA: Efeitos no Peso Induzidos por Proposta de Alteração de Comportamento Alimentar em Hipertensos Medicados Nativos Portugueses e Imigrantes de Origem Africana Acta Med Port 2014:27:331-341

Publicado pela Acta Médica Portuguesa, a Revista Científica da Ordem dos Médicos

Av. Almirante Gago Coutinho, 151

1749-084 Lisboa, Portugal.

Tel: +351218428215

E-mail: submissao@actamedicaportuguesa.com

www.actamedicaportuguesa.com

ISSN:0870-399X | e-ISSN: 1646-0758

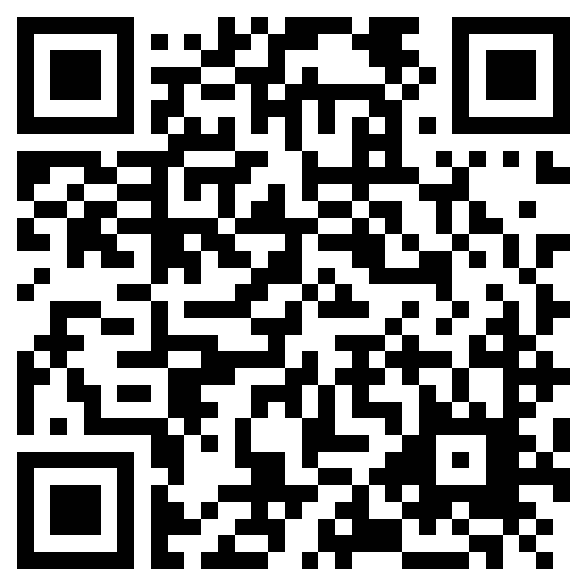

